\title{
Advances in the theory of box integrals
}

\author{
D.H. Bailey* J.M. Borwein ${ }^{\dagger} \quad$ R.E. Crandall ${ }^{\ddagger}$
}

June 25, 2009

\begin{abstract}
Box integrals - expectations $\left\langle|\vec{r}|^{s}\right\rangle$ or $\left\langle|\vec{r}-\vec{q}|^{s}\right\rangle$ over the unit $n$-cube (or $n$ box) - have over three decades been occasionally given closed forms for isolated $n, s$. By employing experimental mathematics together with a new, global analytic strategy, we prove that for $n \leq 4$ dimensions the box integrals are for any integer $s$ hypergeometrically closed in a sense we clarify herein. For $n=5$ dimensions, we show that a single unresolved integral we call $\mathcal{K}_{5}$ stands in the way of such hyperclosure proofs. We supply a compendium of exemplary closed forms that naturally arise algorithmically from this theory.
\end{abstract}

\footnotetext{
${ }^{*}$ Lawrence Berkeley National Laboratory, Berkeley, CA 94720, dhbailey@lbl.gov. Supported in part by the Director, Office of Computational and Technology Research, Division of Mathematical, Information, and Computational Sciences of the U.S. Department of Energy, under contract number DE-AC02$05 \mathrm{CH} 11231$.

${ }^{\dagger}$ School of Mathematical and Physical Sciences, University of Newcastle, Callaghan, NSW 2308, Australia jonathan.borwein@newcastle.edu.au and Faculty of Computer Science, Dalhousie University, Halifax, NS, B3H 2W5, Canada, jborwein@cs.dal.ca. Supported in part by ARC, NSERC and the Canada Research Chair Programme.

${ }^{\ddagger}$ Center for Advanced Computation, Reed College, Portland OR, crandall@reed.edu.
} 


\section{Preliminaries}

We define box integrals ${ }^{1}$ for positive-integer dimension $n$ as expectations of $|\vec{r}|^{s},|\vec{r}-\vec{q}|^{s}$ with the relevant vectors chosen randomly, independent, equidistributed over the unit $n$-cube ${ }^{2}[13]$ :

$$
\begin{aligned}
B_{n}(s) & :=\int_{\vec{r} \in[0,1]^{n}} r^{s} \mathcal{D} \vec{r} \\
& =\int_{0}^{1} \cdots \int_{0}^{1}\left(r_{1}^{2}+\cdots+r_{n}^{2}\right)^{s / 2} d r_{1} \cdots d r_{n} \\
\Delta_{n}(s) & :=\int_{\vec{r}, \vec{q} \in[0,1]^{n}}|\vec{r}-\vec{q}|^{s} \mathcal{D} \vec{r} \mathcal{D} \vec{q} \\
& =\int_{0}^{1} \cdots \int_{0}^{1}\left(\left(r_{1}-q_{1}\right)^{2}+\cdots+\left(r_{n}-q_{n}\right)^{2}\right)^{s / 2} d r_{1} \cdots d r_{n} d q_{1} \cdots d q_{n}
\end{aligned}
$$

Here, $\mathcal{D} \vec{r}$ denotes simply the volume element $d r_{1} d r_{2} \cdots$. As explained in a previous treatment [5], there are physical interpretations:

1. $B_{n}(1)$ is the expected distance of a random point from the origin (or from any fixed vertex) of the $n$-cube;

2. $\Delta_{n}(1)$ is the expected distance between two random points of the $n$-cube;

3. $B_{n}(-n+2)$ is the expected electrostatic potential in an $n$-cube whose origin has a unit charge ${ }^{3}$

4. $\Delta_{n}(-n+2)$ is the expected electrostatic energy between two points in a uniform cube of charged "jellium."

Note that the definitions show immediately that both $\Delta_{n}(2 m)$ and $B_{n}(2 m)$ are rational when $m, n$ are natural numbers. A pivotal, original treatment on box integrals is the 1976 work of Anderssen et al, [1]. There have been interesting modern treatments of the $B_{n}$ and related integrals, as in [7], [11, p.208], [27], [25]. Related material is also to be found in $[16,26]$.

There are other similar entities such as the expected distance between points on distinct sides of a cube or hypercube investigated in $[11, \S 1.7]$ or [7]. We remark that $B_{3}(1)$ is also known as the Robbins constant, after [22].

\footnotetext{
${ }^{1}$ Not to be confused with "box integrals" of particle physics.

${ }^{2}$ We refer to "box integrals" but use the more standard term "cube" or " $n$-cube" for the domain hypercube.

${ }^{3}$ Such statements presume that electrostatic potential in $n$ dimensions is $V(r)=1 / r^{n-2}$, and say $\log r$ for $n=2$; the main issue being that negative powers of $r$ can also have physical meaning.
} 
It is worth noting that the methods of the present paper actually have practical application - for example in biology, of all places. In the work [14] these new box-integral evaluations are being used as one measure of "how random?" is a point cloud. That is, one test of whether brain synapses are sufficiently randomly distributed involves comparison of empirical values $\left\langle|\vec{r}-\vec{q}|^{s}\right\rangle$, where $\vec{r}, \vec{q}$ run over synapse positions, against our present theoretical tabulations of $\Delta_{3}(s)$.

\section{Quadrature formulae for all complex powers}

As in our previous treatment [5], we define two key functions as

$$
\begin{aligned}
b(u) & :=\int_{0}^{1} e^{-u^{2} x^{2}} d x=\frac{\sqrt{\pi} \operatorname{erf}(u)}{2 u}, \\
d(u) & :=\int_{0}^{1} \int_{0}^{1} e^{-u^{2}(x-y)^{2}} d x d y=\frac{-1+e^{-u^{2}}+\sqrt{\pi} u \operatorname{erf}(u)}{u^{2}} .
\end{aligned}
$$

These functions may be used as integration kernels, in the following way. Writing

$$
R^{s}=\frac{1}{\Gamma(-s / 2)} \int_{0}^{\infty} t^{-s / 2-1} e^{-t R^{2}} d t
$$

valid for $\Re(s)<0$, we can integrate formally over $R$ (being a radius, or a separation) in a relevant region, to obtain

$$
\begin{aligned}
B_{n}(s) & =\frac{2}{\Gamma(-s / 2)} \int_{0}^{\infty} u^{-s-1} b^{n}(u) d u, \\
\Delta_{n}(s) & =\frac{2}{\Gamma(-s / 2)} \int_{0}^{\infty} u^{-s-1} d^{n}(u) d u,
\end{aligned}
$$

both representations being valid for a reduced range of complex $s$, namely the range $\Re(s) \in(-n, 0)$. This domain of convergence for the integrals can be inferred from the large- $u$ asymptotic behaviors, see e.g., [2],

$$
\begin{array}{r}
b(u)=\frac{1}{u}+O\left(e^{-u^{2}}\right), \\
d(u) \sim \frac{\sqrt{\pi}}{u}-\frac{1}{u^{2}}+O\left(e^{-u^{2}}\right),
\end{array}
$$

so that having integrand factors $b^{n}, d^{n}$ respectively allows integral convergence for the stated range $\Re(s) \in(-n, 0)$.

What can we say about analytic continuation in $s$ ? We can extend such previous results to all complex $s$, by paying deeper attention to integrand structure. First, we can extend the region for negative $\Re(s)$ arbitrarily, by finding superpositions of terms $b(k u)$ 
or $d(k u)$, for integers $k$, such that the $n$-th powers of the asymptotic forms $(8,9)$ are genuinely of exponential decay.

Second, for positive $\Re(s)$ and a positive integer $K$, we may employ the integral identity

$$
D(\rho, K):=\int_{0}^{\infty} t^{-\rho-1}\left(1-e^{-t u}\right)^{K} d u=\Gamma(-\rho) \sum_{j=1}^{K}\left(\begin{array}{c}
K \\
j
\end{array}\right)(-1)^{j} j^{\rho} .
$$

from which it is straightforward to derive integral representations for all $s$ in the open right half-plane except for the positive even integers.

Theorem 1 [Quadrature formulae for general complex s] In the region with $\Re(s)<0$ we have

$$
B_{n}(s)=\frac{2}{1-2^{n+s}} \frac{1}{\Gamma(-s / 2)} \int_{0}^{\infty} u^{-s-1}\left(b^{n}(u)-2^{n} b^{n}(2 u)\right) d u .
$$

For $\Delta_{n}(s)$ in the same s-region, let $A_{1}:=1$ and consider the unique solution $\left(A_{2}, \ldots, A_{n+2}\right)$ to the linear system

$$
0=\sum_{k=1}^{n+2} \frac{A_{k}}{k^{q}}, \quad q=0,1,2, \ldots, n
$$

Then

$$
\Delta_{n}(s)=\frac{2}{\Gamma(-s / 2)}\left(\sum_{k=1}^{n+2} A_{k} k^{n+s}\right)^{-1} \int_{0}^{\infty} u^{-s-1}\left(\sum_{k=1}^{n+2} A_{k} k^{n} d^{n}(k u)\right) d u
$$

Next, consider the region $\Re(s) \in(0,2 K)$ with $K$ a positive integer and $s$ not an even integer. We have

$$
\begin{aligned}
B_{n}(s) & =\frac{2}{D(s / 2, K)} \int_{0}^{\infty} u^{-s-1} d u \sum_{j=1}^{K}\left(\begin{array}{c}
K \\
j
\end{array}\right)(-1)^{j} b^{n}(u \sqrt{j}) \\
\Delta_{n}(s) & =\frac{2}{D(s / 2, K)} \int_{0}^{\infty} u^{-s-1} d u \sum_{j=1}^{K}\left(\begin{array}{c}
K \\
j
\end{array}\right)(-1)^{j} d^{n}(u \sqrt{j}) .
\end{aligned}
$$

Remark: As for nonnegative even integers $s$ not covered in the above theorem, the definitions $(1,2)$ immediately yield rational values for $B_{n}, D_{n}$ respectively upon symbolic integration.

It is interesting that even though the goal of the above development is to provide practical quadrature formulae, we already have a byproduct: 
Corollary 1 [Pole structure of the box integrals] $B_{n}(s)$, for positive integer $n$, has precisely one pole, namely at $s=-n . \Delta_{n}(s)$, on the other hand, always has precisely $(n+1)$ poles, located at $s=-2 n,-2 n+1, \ldots,-n$.

Proof: Clearly, by the original definitions (1) and (2), $B_{n}(s), \Delta_{n}(s)$ are both finite for nonnegative $\Re(s)$ so it suffices to look at negative $\Re(s)$ in any case. For $B_{n}(s)$, relation (11) has a pole factor at $s=-n$.

As for $\Delta_{n}(s)$, the solution $\left(A_{k}\right)$ to the system of equations is unique since the matrix with entries $(-1)^{k} /(k+1)^{j-1}$ for $1 \leq k, j \leq n$ is nonsingular with determinant $(-1)^{\lfloor n / 2\rfloor} \prod_{i=1}^{n-1} \frac{i !}{(n+1) !}$ (see sequence A002109 in Sloane's Online Encyclopedia of Integer Sequences). Poles of $\Delta_{n}$ force the prefactor $\sum_{k=1}^{n+2} A_{k} k^{n+s}$ to vanish exactly when $n+s \in[-n, 0]$, thus giving a total of $(n+1)$ poles on the negative real $s$-axis. It remains to check that $\sum_{k=1}^{n+2} A_{k} k^{n+s}$ has no other zeros. This runs as follows. Consider the more general exponential sum

$$
\Phi_{N}(t):=\sum_{j=1}^{N} \alpha_{j} \beta_{j}^{t}=1
$$

for arbitrary real constants $\alpha_{j}$ and positive $\beta_{j}$. If $\Phi_{N}(t)=1$ has more than $N$ solutions then Rolle's theorem and some manipulation will produce a derived system with at least $N$ solutions and one less term. We are done, since when $N=1$ this is impossible. QED

By employing the quadrature options embodied in Theorem 1, we have been able to calculate various $B_{n}(s), \Delta_{n}(s)$ values to extreme precision. ${ }^{4}$

One may also use the quadrature prescription to create contiguous plots of box integrals, as in Figure 1. Referring to Figure 1, note that both plots show the "natural" pole at $s=-2$, where the standard expectation integral diverges for $n=2$ dimensions. However, we see that $\Delta_{2}$ also has poles at $s=-3,-4$ as in Corollary 1 .

\section{Quadrature experiments}

We employed the quadrature schema of Theorem 1 to effect extreme-precision values. The values shown to 50-digit accuracy in Section 7 were computed to over 400 digits. We might mention here that in fact this research began in earnest with the discovery, using the PSLQ integer relation algorithm [15],[8], that the extreme-precision numerical value we were able to compute for $\Delta_{3}(-1)$, namely

$$
\Delta_{3}(-1)=1.88231264438966016010560083886836758785246288031070 \ldots
$$

\footnotetext{
${ }^{4}$ As in previous works, by "extreme precision" we mean, loosely speaking, enough precision to resolve a given entity into fundamental (or at least previously studied) constants; in the modern era - the age of, say, LLL and PSLQ methods - this usually means precision to hundreds to thousands of decimal digits.
} 
was (experimentally) given by the formula

$$
\begin{aligned}
\Delta_{3}(-1) \stackrel{?}{=} & \frac{2}{5}+\frac{2}{5} \sqrt{2}-\frac{4}{5} \sqrt{3}-\frac{2}{3} \pi-6 \log 2+2 \log (1+\sqrt{2}) \\
& +12 \log (1+\sqrt{3})-4 \log (2+\sqrt{3}) .
\end{aligned}
$$

Soon we were able to prove this evaluation. ${ }^{5}$ Upon further experimentation and analytic discoveries, we realized that not only do some $B_{n}$ for various $n$ have constants in common-such as log-surds, $\pi$, and so on-but the $\Delta_{n}$ constants also have many constants in common with themselves and the $B_{n}$. Such experimentation/detection moved us to find algebraic relations between the various entities, as we describe in this paper.

Our numerical approach was to utilize the ARPREC arbitrary precision software package [10] to implement the four formulae of Theorem 1, namely (11), (13), (14) and (15), together with the definitions of the functions $b(u), d(u)$ and $D(\rho, K)$ defined by formulae (4), (3) and (10). As stated in Theorem 1, these formulae are valid for all $s$ except for positive even integers. For positive even arguments the integration in $(1,2)$ is trivial and the relevant box integral is rational, obtainable quickly with symbolic processing. ${ }^{6}$

Computation of $b(u)$ is entirely straightforward, once one has in hand an implementation of the erf function (see [2, pg. 297-298]).

Computation of $d(u)$ is complicated by the fact that for small arguments, cancellation of terms in the numerator typically results in severe relative numerical error. This can be ameliorated by employing the Taylor series for $d(u)$ :

$$
\begin{aligned}
d(u) & =1-\frac{u^{2}}{6}+\frac{u^{4}}{30}-\frac{u^{6}}{168}+\frac{u^{8}}{1080}-\frac{u^{10}}{7920}+\frac{u^{12}}{65520}-\cdots \\
& =\sum_{n=0}^{\infty} \frac{(-1)^{n} u^{2 n}}{(2 n+1) \cdot(n+1) !}
\end{aligned}
$$

Computation of the $A_{k}$ coefficients was achieved by employing an arbitrary precision version of the well-known Linpack program for solving linear equations via LU decomposition $[20]$.

The four formulae (11), (13), (14) and (15) of Theorem 1 themselves involve significant numerical difficulties, as they involve near-cancellation of terms for small arguments. Nonetheless we were able to compute the $B_{n}(s)$ and $D_{n}(s)$ to approximately 470-digit

\footnotetext{
${ }^{5}$ That is to say, the $\stackrel{?}{=}$ became $=$. In the present work, we use $=$ to mean rigorously proven, even though some of our more recondite expressions were first found in the $\stackrel{?}{=}$ sense, then later proven.

${ }^{6}$ Mathematica and Maple were both used quite heavily for much of this paper's development, and most of the work was symbolic. We have endeavored to check all results numerically, even after obtaining exact, symbolic forms. That being said, elsewhere in this paper we have for efficiency eschewed comments about which computer algebra system was used where.
} 
accuracy, utilizing 500-digit working precision, by using Gaussian quadrature, after splitting the integral into the intervals $(0,1)$ and $(1, \infty)$, and applying the simple substitution $w=1 / u$ on the second integral.

We should add here that tanh-sinh quadrature [24], which we have used in numerous other studies of this sort, was not needed in this case, because each of the integrand functions in Theorem 1 are regular on the intervals of integration. In addition, the tanhsinh scheme suffers here from the cancellation difficulties mentioned above, since it relies on evaluating the function very close to endpoints of the interval.

\section{Analytic continuation for the $B_{n}$}

In our previous work [5] we demonstrated that the $B_{n}$ expectations can always be reduced by at least one dimension; for example, via vector-field algebra, $B_{n}$ can be obtained from an integral over the $(n-1)$-dimensional unit cube, like so for $n>1$ :

$$
B_{n}(s)=\frac{n}{n+s} \int_{\vec{r} \in[0,1]^{n-1}}\left(1+r^{2}\right)^{s / 2} \mathcal{D} \vec{r} .
$$

Note that already with this reduction we see that $B_{n}$ has a single, simple pole at $s=-n$, as the integral exists for all complex $s$.

With the advantage of hindsight, we define right now a general and quite useful function

$$
C_{n, k}(s, a):=\int_{\vec{r} \in[0,1]^{n}} r_{1} r_{2} \cdots r_{k}\left(a+r^{2}\right)^{s / 2} \mathcal{D} \vec{r}
$$

and we also define $C_{0,0}(s, a):=a^{s / 2}$. In this way we can begin developing relations for the $B_{n}$, as follows:

$$
\begin{aligned}
& B_{1}(s)=\frac{1}{s+1} C_{0,0}(s, 1)=\frac{1}{s+1} \\
& B_{2}(s)=\frac{2}{2+s} C_{1,0}(s, 1)=\frac{2}{2+s}{ }_{2} F_{1}\left(\frac{1}{2},-\frac{s}{2} ; \frac{3}{2} ;-1\right), \\
& B_{3}(s)=\frac{3}{3+s} C_{2,0}(s, 1)=\frac{6}{(3+s)(2+s)} \int_{0}^{\pi / 4}\left(\left(1+\sec ^{2} t\right)^{s / 2+1}-1\right) .
\end{aligned}
$$

Note that the apparent pole at $s=-2$ for $B_{3}(s)$ is specious; the $1 /(s+2)$ factor cancels in this case, to yield

$$
B_{3}(-2)=3 \int_{0}^{\pi / 4} \log \left(1+\sec ^{2} t\right) d t .
$$

It will be important later to observe a recurrence for the $C$-function in (25), which recurrence will also play a major role in our later development of the $\Delta_{n}$ box integrals. 
For $n=4$ and higher dimensions, such relations for the $B_{n}$ rapidly become unwieldy. Take $n=4$, and note that $C_{3}(s, 1)$ is a 3 -dimensional integral which yields, after integrating first in polar coordinates $(r, \phi)$ to handle two of the dimensions,

$$
\begin{aligned}
B_{4}(s) & =\frac{4}{4+s} C_{3,0}(s, 1) \\
& =\frac{8}{4+s} \frac{1}{2+s} \int_{0}^{\pi / 4} d \phi \int_{0}^{1}\left(\left(1+\sec ^{2} \phi+z^{2}\right)^{s / 2+1}-\left(1+z^{2}\right)^{s / 2+1}\right) d z .
\end{aligned}
$$

Again the specious pole factor for $s=-2$ cancels, giving the special case

$$
B_{4}(-2)=2 \int_{0}^{\pi / 4} d \phi \int_{0}^{1}\left(\log \left(1+\sec ^{2} \phi+z^{2}\right)-\log \left(1+z^{2}\right)\right) d z .
$$

Though we are left with some stultifying integrals, the complete continuations above for $B_{1}, B_{2}, B_{3}, B_{4}$ do indicate some powerful experimental-mathematical pathways. Later we shall be able to build upon previous literature results by adding new closed forms to our knowledge.

There is another form of analytic relation also derived previously [5], namely

$$
B_{n}(s)=\frac{n^{s}}{n+s} \sum(-s / 2)_{k}\left(\frac{2}{n}\right)^{k} \beta_{n-1, k},
$$

where the $\beta$ coefficients arise from the $b$ function, implicitly as

$$
\left(b(u) e^{t u^{2}}\right)^{n}=: \sum_{k \geq 0} \beta_{n, k} 2^{k} u^{2 k} .
$$

and $(a)_{k}=\Gamma(a+k) / \Gamma(a)$ is the Pochhammer symbol.

The $\beta$ numbers enjoy the relations (here, $d !$ ! means $1 \cdot 3 \cdots d$ for odd-positive integer $d)$ :

$$
\begin{aligned}
\beta_{n, k} & =\sum_{k_{1}+\ldots k_{n}=k} \frac{1}{\left(2 k_{1}+1\right) ! !} \cdots \frac{1}{\left(2 k_{n}+1\right) ! !}, \\
\beta_{n, k} & =\sum_{j=0}^{k} \frac{1}{(2 k+1) ! !} \beta_{n-1, k-j}, \\
(1+2 k / n) \beta_{n, k} & =\beta_{n, k-1}+\beta_{n-1, k},
\end{aligned}
$$

with the recursions here ignited by $\beta_{0, k}:=\delta_{0, k}$ and $\beta_{n, 1}=n / 3$. Here !! denotes the double factorial function: $m ! !=m(m-2)(m-4) \cdots 3 \cdot 1$ if $m$ is odd, and $m ! !=m(m-2)(m-$ 4) $\cdots 4 \cdot 2$ if $m$ is even.

The main point is, for $n>1$ the sum (29) is linearly absolutely convergent for all complex $s$. (The summand decay factor is essentially $(1-1 / n)^{k}$.) Accordingly, the series 
gives the analytic continuation over the entire $s$-plane. This series representation can be used computationally, either in place of the quadrature formulae, or as an extremeprecision check on same. The analytic series does have one advantage over the quadrature formalism: One need not break the plane by polarity in $\Re(s)$; the series (29) always converges (and there is the correct single-pole factor $1 /(n+s)$ ).

\section{Theory of hyperclosure and of the $C$-functions}

We have seen that the box integrals $B_{n}$ are expressible in terms of $C$-functions. It will turn out that there exist recurrence relations between $C$-functions; this leads eventually to relations between $\Delta_{n}$ and $B_{n}$ values, and closed forms in many cases.

To quantify for our purposes what is a closed form, we first establish ${ }^{7}$

Definition 1 [Ring of hyperclosure] We define a hypergeometric evaluation in a classical sense-to be a complex number $\sum_{n \geq 0} a_{n} z^{n}$ where $z$ is algebraic, $a_{0}$ is rational-real, and generally $a_{n+1}=r(n) a_{n}$ where $r$ is a fixed rational-polynomial function (ratio of two polynomials each with integer coefficients). We then contemplate the ring of hyperclosure, generated by all hypergeometric evaluations under ordinary operators $(\cdot,+)$. An element of this ring is said to be hypergeometrically closed (hyperclosed for short). We also deem $\infty$ to be hyperclosed (so that hyperclosure not be denied at poles).

As examples, any rational (a sum with one term, say $r:=0$ ) is hyperclosed, and since

$$
\sqrt{2}={ }_{2} F_{1}\left(-\frac{1}{2}, 1,1 ;-1\right), \quad \pi=4{ }_{2} F_{1}\left(\frac{1}{2}, 1,3 / 2,-1\right), \quad \log (1+z)=z_{2} F_{1}(1,1,2 ;-z),
$$

it follows that combinations such as $\sqrt{2}+\pi, \pi \sqrt{2}, \pi^{10} \log ^{7} 2, \frac{1}{3} \log (1+\sqrt{2})$ are all hyperclosed. Relevant to the present work is the fact of the Lerch transcendent

$$
\Phi(z, s, a):=\sum_{n \geq 0} \frac{z^{n}}{(n+a)^{s}},
$$

being hyperclosed for algebraic $z$, integer $s$, and rational $a$. Thus, we shall have hyperclosure for any polylogarithm $\operatorname{Li}_{s}(z):=\sum_{n \geq 1} z^{n} / n^{s}$ for integer $s$ and algebraic $z$, so perforce for the Lewin arctan integral

$$
\operatorname{Ti}_{2}(z):=\sum_{n \geq 0}(-1)^{n} \frac{z^{2 n+1}}{(2 n+1)^{2}}=\frac{z}{4} \Phi\left(-z^{2}, 2,1 / 2\right),
$$

\footnotetext{
${ }^{7}$ It has occurred to the present authors that the age-old notion of "closed form" might be addressable, as a separate research program, vis such as our Definition 1. It is a pleasant task to be able to talk about closed forms with some semblance, at least, of rigor.
} 
and the Clausen function

$$
\mathrm{Cl}_{2}(z):=\sum_{n \geq 1} \frac{\sin (n z)}{n^{2}}
$$

Our box-integral tables have hyperclosed entries, except where "dangling integrals" remain when dimension $n>4$. (Although we admit such unresolved integrals may still belong to the ring.)

An interesting sidelight looms here: What numbers are not hyperclosed? Certainly we need such numbers to exist, lest our entire research program of finding hyperclosed expressions be a vacuous exercise. Given the algebraic character of parameter $z$ in Definition 1 , and the constraint that the succession ratio $r(n)$ be rational-polynomial, together with the fact of all group generations under $(\cdot,+)$ yielding finite strings, we conclude that the ring of hyperclosure is countably infinite. Therefore: Almost all complex numbers are not hyperclosed. Equivalently, the ring of hyperclosure is a null set in the complex plane. As often happens in such analyses, we are stultified by the question: "If non-hyperclosed numbers are so overwhelmingly abundant, what is an example of such a number?" Well, we do not presently know a single such number. Perhaps $\pi^{\pi}$ is not hyperclosed, but such questions loom Hilbertian in their evident profundity. ${ }^{8}$

It is evident that the lowest-lying $C$-functions allow direct hypergeometric evaluation, so that $B_{1}(s), B_{2}(s)$ are hyperclosed for any integer $s$, but that already for $C_{2,0}$ we run into some difficulty with the integration. Happily, there are some powerful connection formulae that we prove presently, starting with

Theorem 2 [Convergent series for $C$-functions] For all complex s, integer $n>1$, and $\Re(a)>0$ we have

$$
C_{n, 0}(s, a)=(n+a)^{s / 2} \sum_{k \geq 0}\left(\frac{2}{n+a}\right)^{k}(-s / 2)_{k} \beta_{n, k}
$$

which expansion being linearly convergent with $k$-th summands being $O\left(((n-1) /(n+a))^{k}\right)$.

Proof: This expansion can be established using the same methods as for expansion (29), which is the case $a=1$ as shown in [5].

QED

And now for the recurrence:

\footnotetext{
${ }^{8}$ Such a radical disconnect between theory and knowledge occurs elsewhere; e.g., in the study of normal numbers. Though almost all real numbers are (absolutely) normal, only artificially constructed normals are known.
} 
Theorem 3 [Fundamental relations for $C$-functions] Special instances of the $C$ function are

$$
\begin{aligned}
& C_{n, 0}(s, 0)=B_{n}(s), \\
& C_{0,0}(s, a):=a^{s / 2}, \\
& C_{n, 0}(s, 1)=\frac{n+s+1}{n+1} B_{n+1}(s) .
\end{aligned}
$$

More generally, for all complex $s$, positive integer $n$, and positive real a we have

$$
a s C_{n, 0}(s-2, a)=(s+n) C_{n, 0}(s, a)-n C_{n-1,0}(s, a+1) .
$$

Another recurrence, involving now the second index on $C$, is, for positive integers $a, k$,

$$
s C_{n, k}(s-2, a)=C_{n-1, k-1}(s, a+1)-C_{n-1, k-1}(s, a) .
$$

Proof: The recurrence (39) is proved by invoking $\beta$-relation (31) to show the right-hand side here in the theorem is $2 a\left(\partial C_{n, 0}(s, a) / \partial a\right)$ which equals the left-hand side. The recurrence (40) is easier, requiring simple integration by parts in the integral representation (22) for $C$.

QED

We are now prepared to establish a central result that will lead to a host of closed forms, which result being:

Theorem 4 For any integer $s$ and positive integer a, all the numbers

$$
C_{n, 0}(s, a):=\int_{\vec{r} \in[0,1]^{n}}\left(a+r^{2}\right)^{s / 2} \mathcal{D} \vec{r}
$$

for $n=0,1,2$ are hyperclosed.

Remark: As with other parts of the present treatment, we not only prove hyperclosure, but do this constructively, so that an algorithm for achieving closed forms is intrinsic to the proof.

Proof: $\quad C_{0,0}(s, a):=a^{s / 2}$ and $C_{1,0}(s, a)=a^{s / 2}{ }_{2} F_{1}\left(\frac{1}{2},-s / 2 ; 3 / 2,-1 / a\right)$ are clearly hyperclosed. So we turn our attention to $C_{2,0}$ : For nonnegative even integer $s$, it is evident that the defining integral for $C_{2,0}$ yields rational values. Consider, then, a single evaluation for negative even $s$, where we employ polar coordinates in the defining 2-dimensional integral to obtain

$$
\begin{aligned}
C_{2,0}(-2, a) & =\int_{0}^{\pi / 4} d t\left(\log \left(a+\sec ^{2} t\right)-\log a\right) \\
& =-G+\operatorname{Ti}_{2}\left(\frac{2+a-2 \sqrt{1+a}}{a}\right)+\frac{\pi}{2} \log (1+\sqrt{1+a})-\frac{\pi}{4} \log a,
\end{aligned}
$$


where $G$ is the Catalan constant and $\mathrm{Ti}_{2}$ is the Lewin inverse-tangent integral; see (32, 33). (Note that $G=\operatorname{Ti}_{2}(1)=\sum_{n \geq 0}(-1)^{n} /(2 n+1)^{2}$.) Clearly $C_{2,0}(-2, a)$ is hyperclosed for positive algebraic $a$. Now, in recurrence (39) with $n=2$, there is a term $C_{1,0}(s, a+1)$ which we have seen to be hyperclosed. The recurrence thus reveals that $C_{2,0}(-s, a)$ is hyperclosed for all negative even integers $s$.

It remains to handle all odd integers $s$. We observe another "ignition value"

$C_{2,0}(1, a)=-\frac{1}{3} a^{3 / 2} \tan ^{-1}\left(\frac{1}{\sqrt{a^{2}+2 a}}\right)+\frac{1}{6}(3 a+1) \log \left(\frac{a+2 \sqrt{a+2}+3}{a+1}\right)+\frac{1}{3} \sqrt{a+2}$,

and this again drives the recurrence (39) to yield hyperclosure for any odd $s$.

QED

\section{Hyperclosure and the $B_{n}$}

Theorem 5 [General interrelations for the $B_{n}$ ] For integer $n>1$ and all complex $s$, we have a recurrence

$$
(n+s)(n+s-1) B_{n}(s)=s(n+s-2) B_{n}(s-2)+n(n-1) C_{n-2,0}(s, 2),
$$

and a higher-order variant

$$
\begin{aligned}
(n+s)(n+s-1)(n+s-3) B_{n}(s)= & s(s-2)(n+s-4) B_{n}(s-4)+ \\
& s n(n-1) C_{n-2,0}(s-2,2)+ \\
& (n+s-3) n(n-1) C_{n-2,0}(s, 2) .
\end{aligned}
$$

In addition, the residue $\operatorname{Res}_{n}$ of the unique pole at $B_{n}(-n)$ is given for all positive integers $n$ by $\operatorname{Res}_{1}=1$ and for $n>1$,

$$
\operatorname{Res}_{n}=-2 n B_{n}(-n-2)-n(n-1) C_{n-2,0}(-n, 2) .
$$

Finally, we have a special analytic-continuation value valid for all positive integers $n$ :

$$
B_{n}(-n-1)=-n C_{n-2}(1-n, 2) \text {. }
$$

Remark: In cases such as $s=-n$ where a pole is involved, an expression $(n+s) B_{n}(s)$, on the face of it $0 \cdot \infty$, is to be interpreted as the residue $\operatorname{Res}_{n}$ at the relevant pole. It turns out that the second recurrence allow us to traverse $B_{n}$-poles. Both the residue relation and the special analytic-continuation relation follow from the first recurrence.

Proof: The first $B_{n}, C$ recurrence follows from the combination of proven relations (38) and (39). The second, higher-order recurrence is obtained by double application of the first recurrence.

QED 
Theorem 6 [Hyperclosure for specific $B_{n}$ ] For any integer s, each of:

$$
B_{1}(s), B_{2}(s), B_{3}(s), B_{4}(s)
$$

is hyperclosed. Moreover, the residues $\operatorname{Res}_{n}$ at the four poles $B_{n}(-n)$ for $n=1,2,3,4$ are also hyperclosed.

Remark: Again the proof following is constructive; i.e., gives rise to an immediate algorithm for generating closed forms.

Proof: From Theorem 4 it is immediate that $B_{m}(s)=\frac{m}{m+s} C_{m-1,0}(s, 1)$ is hyperclosed for $m=1,2,3$. So we turn to $B_{4}$, and observe that for nonnegative even integer $s$, the value $B_{4}(s)$ is rational. From Theorem 5 we have two recurrences

$$
\begin{aligned}
(3+s)(4+s) B_{4}(s) & =s(s+2) B_{4}(s-2)+12 C_{2,0}(s, 2), \\
(3+s)(4+s)(1+s) B_{4}(s) & =s^{2}(s-2) B_{n}(s-4)+12 s C_{2,0}(s-2,2)+12(s+1) C_{2,0}(s, 2) .
\end{aligned}
$$

Inspection of these reveals that if we establish hyperclosure for $B_{4}(-1)$ and $B_{4}(-2)$, say, then we know all $B_{4}$ (integer) to be hyperclosed via Theorem 4 . (The first recurrence above is ignited by $B_{4}(-1)$, for all $B_{4}$ (odd integer), while the second recurrence propagates eveninteger arguments across the pole at $s=-4$.) To these ends, we observe

$$
B_{4}(-1)=2 \log (3)-\frac{2}{3} G+2 \operatorname{Ti}_{2}(3-2 \sqrt{2})-\sqrt{8} \arctan \left(\frac{1}{\sqrt{8}}\right),
$$

and

$$
B_{4}(-2)=\pi \log (2+\sqrt{3})-2 G-\frac{\pi^{2}}{8} .
$$

Starting with (27) and the evaluation of the jellium value $J_{4}$ given in our previous paper [5], the proof of (50) reduces to showing

$$
\int_{0}^{1} \frac{\log \left(3+s^{2}\right)}{1+s^{2}} d s=\frac{\pi}{4} \log (2+\sqrt{3})+\frac{\pi}{4} \log (2)-\mathrm{Ti}_{2}(-2+\sqrt{3})-G .
$$

Using A2.2(3) in [19] this becomes

$$
\int_{0}^{1} \frac{\log \left(3+s^{2}\right)}{1+s^{2}} d s+\frac{\pi}{6} \log (2+\sqrt{3})-\frac{\pi}{4} \log (2)-\frac{1}{3} G=0 .
$$

Now substituting $s=\tan \theta$ yields an expression which computer algebra evaluates as

$$
\frac{4}{3} G+\frac{\pi}{3} \log (2+\sqrt{3})-\mathrm{Ti}_{2}(2+\sqrt{3})-\mathrm{Ti}_{2}(2-\sqrt{3})=0 .
$$


This is easily confirmed using [19, A2.2(3),(4)]. The proof of (49) is similarly completed; said proof reduces to showing (72) noted below.

The hyperclosure of the $\operatorname{Res}_{n}$ now follows immediately from the residue recursion formula given in Theorem 5 .

QED

Another approach to recursions for $B_{n}, \Delta_{n}$ as a function of $s$ (for all positive integer $n$ ) is via the linear differential equations satisfied by $b$ and $d$ see [12] and [11, p. 270]. This leads, for example, to an implementable 5 -term recursion for $B_{4}(s)$ in terms of $B_{4}(s-2), B_{4}(s-4), B_{4}(s-6), B_{4}(s-8)$ with coefficients polynomial in $s$. (Note that tighter recursions are possible, as in Theorem 9 for $n=5,6$ dimensions, provided we only demand that terms be hyperclosed.)

\section{Hyperclosure and the $\Delta_{n}$}

On the face of it, our definition (2) of the second box integral, repeated here:

$$
\Delta_{n}(s):=\int_{\vec{r}, \vec{q} \in[0,1]^{n}}|\vec{r}-\vec{q}|^{s} \mathcal{D} \vec{r} \mathcal{D} \vec{q}
$$

would appear to present a $(2 n)$-dimensional integration problem. But this is not so; in fact we have quite generally

$$
\Delta_{n}(s)=2^{n} \int_{\vec{r} \in[0,1]^{n}} r^{s} \prod_{k=1}^{n}\left(1-r_{k}\right) \mathcal{D} \vec{r} .
$$

This reduction from $2 n$ to $n$ dimensions can be derived in various ways. Perhaps the most intuitive is to look at the probability density of a coordinate difference $x=r_{k}-q_{k}$, which turns out to be a triangle distribution with base $[-1,1]$ and height 1 at the origin-centered apex; i.e., the density is $1-|x|$, and the new integral form follows.

It is interesting that (51) has "leading term" $2^{n} B_{n}(s)$ arising from the 1 component of the integrand's product, indicating heuristically that $\Delta_{n}$ and $B_{n}$ should somehow be analytic relatives. Indeed, one can carry such an idea much further, to end up with powerful analytic expressions for $\Delta_{n}(s)$. Simple symmetries of the integrand in (51) reveal that

$$
\Delta_{n}(s)=2^{n} \sum_{k=0}^{n}\left(\begin{array}{l}
n \\
k
\end{array}\right)(-1)^{k} C_{n, k}(s, 0) .
$$

On the other hand, we have the recursion (40) that may be used to decrement the both indices $n, k$ on $C_{n, k}$. Thus we have the intriguing principle that $\Delta_{n}$ can be formally expressed in terms of $C_{m, 0}$ functions, where $m \leq n$. But there is more:

Some thought in regard to relations $(38,39,44)$ reveals that the relevant $C_{m, 0}$ values lead back to the $B_{n}$ box integrals, so that $\Delta_{n}$ can be formally expressed in terms of the $B_{n}$. The summary result is: 
Theorem $7\left[\Delta_{n}\right.$ as an analytic superposition of $\left.B_{2}, \ldots, B_{n}\right]$ For arbitrary positive integer $n$ and any complex $s$, we have an analytic superposition

$$
\Delta_{n}(s)=E_{n}(s)+\sum_{m=2}^{n} \sum_{j=1}^{m} R_{m, j}(s) B_{m}(s+2 n-2 j),
$$

where $E_{n}$ is an elementary function and each $R_{m, j}$ is a rational-polynomial function.

Remark: Thus $\Delta_{n}$ is in general a superposition of $(n+2)(n-1) / 2$ different $B$ terms.

Proof: This result follows from the combinatorics embodied in relations $(52,38,39$, 44).

QED

Let us now give some entirely general analytic $\Delta_{n}$ evaluations; first the easy case $n=1$, for which the sum in Theorem 7 is empty and we have a simple, elementary function:

$$
\Delta_{1}(s)=2 \int_{0}^{1}(1-x) x^{2} d x=\frac{2}{1+s}-\frac{2}{2+s} .
$$

Note the two poles, as we expect from previous analysis. Then from the combinatorics leading up to Theorem 7 we obtain

$$
\Delta_{2}(s)=8 \frac{(3+s) 2^{\frac{s}{2}+1}+1}{(s+2)(s+3)(s+4)}+4 B_{2}(s)-\frac{4(s+4)}{s+2} B_{2}(s+2) .
$$

Note now the existence of three poles (at $s=-4,-3,-2)$. Going yet deeper into the combinatorics, we have

$$
\begin{aligned}
\Delta_{3}(s)= & \frac{24\left(-3^{\frac{s}{2}+2} s+2^{\frac{s}{2}+3} s-53^{\frac{s}{2}+2}+52^{\frac{s}{2}+3}+1\right)}{(s+2)(s+4)(s+5)(s+6)}+\frac{24}{s+2} B_{2}(s+2)- \\
& \frac{24(s+6)}{(s+2)(s+4)} B_{2}(s+4)+8 B_{3}(s)-\frac{12(s+5)}{s+2} B_{3}(s+2)+ \\
& \frac{4(s+6)(s+7)}{(s+2)(s+4)} B_{3}(s+4) .
\end{aligned}
$$

There are some difficulties attendant on this $\Delta_{3}$ form; namely, the apparent pole at $s=-2$ is specious. In order to get such as the $\Delta_{3}(-2)$ closed form in our tables, one needs to take a careful limit $s \rightarrow-2$. Note also that $\Delta_{3}$ pole at $s=-3$ is caused exclusively by the $8 B_{3}(s)$ term itself. Also, one needs the residue formula from Theorem 5 for the $B_{3}(s+4)$ term at $s=-7$. 
At the next level we obtain the complete analytic continuation for $\Delta_{4}$, in terms of $B_{2}, B_{3}, B_{4}$, :

$$
\begin{aligned}
\Delta_{4}(s)= & \frac{64\left(\left(3 \cdot 2^{\frac{s}{2}+3}+2^{s+6}-3^{\frac{s}{2}+4}\right) s+21 \cdot 2^{\frac{s}{2}+3}-7 \cdot 3^{\frac{s}{2}+4}+7 \cdot 2^{s+6}+1\right)}{(s+2)(s+4)(s+6)(s+7)(s+8)}+ \\
& \frac{96}{(s+2)(s+4)} B_{2}(s+4)-\frac{96(s+8)}{(s+2)(s+4)(s+6)} B_{2}(s+6)+\frac{64}{s+2} B_{3}(s+2)- \\
& \frac{96(s+7)}{(s+2)(s+4)} B_{3}(s+4)+\frac{32(s+8)(s+9)}{(s+2)(s+4)(s+6)} B_{3}(s+6)+16 B_{4}(s)- \\
& \frac{88(s+6)}{3(s+2)} B_{4}(s+2)+\frac{8(s+8)(6 s+43)}{3(s+2)(s+4)} B_{4}(s+4)- \\
& \frac{8(s+8)(s+9)(s+10)}{3(s+2)(s+4)(s+6)} B_{4}(s+6) .
\end{aligned}
$$

Again the apparent pole at $s=-2$ is specious, so one must work out another logarithmic limit in that case. For $s=-9,-10$ we may use the known residues of the $B$-poles. (The poles at $s=-4,-5,-6,-7,-8$ do exist; e.g. the term with $B_{3}(s+2)$ has a pole at $s=-5$.)

Theorem 8 [Hyperclosure for specific $\left.\Delta_{n}\right]$ For any integer s, each of:

$$
\Delta_{1}(s), \Delta_{2}(s), \Delta_{3}(s), \Delta_{4}(s)
$$

is hyperclosed.

Remark: Thus, with Theorem 6 , we now know that $B_{1,2,3,4}$ and $\Delta_{1,2,3,4}$ are all hyperclosed at every integer argument. Our tables show exemplary closed forms.

Proof: From the specific instances of Theorem 7 displayed above for $n=1,2,3,4$, the hyperclosure of the relevant $B_{n}$ (integer), together with special limits taken for the problematic cases $\Delta_{3}(-2), \Delta_{3}(-7), \Delta_{4}(-2), \Delta_{4}(-9), \Delta_{4}(-10)$ completes the proof. QED

\section{Difficulties in $n \geq 5$ dimensions}

We have not been able to fully obtain hyperclosure for $n=5$ because we cannot completely handle the term $C_{3,0}$ that appears in the recursions at that level. That said, we can show hyperclosure for odd integers and report significant progress for the even cases. To begin with we can prove that

$$
\begin{aligned}
B_{5}(1)= & \frac{1}{6} \sqrt{5}+\frac{1}{360} \pi^{2}-\frac{4}{3} \sqrt{3} \arctan \left(\frac{1}{\sqrt{15}}\right)+\frac{10}{3} \log \left(\frac{\sqrt{5}+1}{2}\right) \\
& +\frac{7}{18}\left\{\mathcal{K}_{1}-\frac{\pi}{2} \log (2+\sqrt{3})\right\}
\end{aligned}
$$


and

$$
B_{5}(-1)=\frac{5}{3}\left\{\mathcal{K}_{1}-\frac{\pi}{2} \log (2+\sqrt{3})\right\}+\frac{1}{48} \pi^{2}-5 \sqrt{6} \arctan \left(\frac{1}{\sqrt{15}}\right)+5 \log \left(\frac{\sqrt{5}+1}{2}\right) .
$$

where $\mathcal{K}_{1}$ is a certain definite integral that has been resolved; see next section.

More generally we have

$$
\begin{aligned}
(2+s)(4+s) B_{5}(s)= & 4 \int_{0}^{\pi / 4} \int_{0}^{\pi / 4} \int_{0}^{1}\left(\sec ^{2}(a)+\sec ^{2}(b)+z^{2}\right)^{2+s / 2} d z d a d b- \\
& 2 \pi \int_{0}^{\pi / 4}{ }_{2} F_{1}\left(\frac{1}{2},-2-\frac{s}{2} ; \frac{3}{2} ;-\cos ^{2}(b)\right) \sec ^{4+s}(b) d b+\frac{1}{4} \frac{\pi^{2}}{5+s} .
\end{aligned}
$$

For integer $s$ the integral in $z$ can be performed symbolically and the hypergeometric function evaluated. This reduces $B_{5}(s)$ to a double integral expression for $s=-6$; and also for $s=-2,-4$ on applying l'Hôpital's rule.

The values in Table 4 then can be pieced together from the tools provided and knowledge of integrals $\mathcal{K}_{4}, \mathcal{K}_{5}$ as discussed in the next section (the latter of these two integrals remains unresolved as of the present writing).

Finally, as with lower dimensions, we can supply the following superposition formula for $\Delta_{5}$ in terms of $B_{n}$ with $2 \leq n \leq 5$,

$$
\begin{aligned}
\Delta_{5}(s) & =160 \frac{1+(9+s)\left(2^{6+s / 2}+2^{10+s}-5^{4+s / 2}-2 \cdot 3^{5+s / 2}\right)}{(2+s)(4+s)(6+s)(8+s)(9+s)(10+s)} \\
& +\frac{320}{(2+s)(4+s)(6+s)} B_{2}(6+s)-\frac{320(10+s)}{(2+s)(4+s)(6+s)(8+s)} B_{2}(8+s) \\
& +\frac{320}{(2+s)(4+s)} B_{3}(4+s)-\frac{480(9+s)}{(2+s)(4+s)(6+s)} B_{3}(6+s) \\
& +\frac{160(10+s)(11+s)}{(2+s)(4+s)(6+s)(8+s)} B_{3}(8+s) \\
& +\frac{160}{2+s} B_{4}(2+s)-\frac{880}{3} \frac{(8+s)}{(2+s)(4+s)} B_{4}(4+s)+\frac{80}{3} \frac{(10+s)(55+6 s)}{(2+s)(4+s)(6+s)} B_{4}(6+s) \\
& -\frac{80}{3} \frac{(10+s)(11+s)(12+s)}{(2+s)(4+s)(6+s)(8+s)} B_{4}(8+s) \\
& +32 B_{5}(s)-200 \frac{(7+s)}{6+3 s} B_{5}(2+s)+\frac{4}{3} \frac{(9+s)(291+35 s)}{(2+s)(4+s)} B_{5}(4+s) \\
& -\frac{8}{3} \frac{(10+s)(11+s)(47+5 s)}{(2+s)(4+s)(6+s)} B_{5}(6+s)+\frac{4}{3} \frac{(10+s)(11+s)(12+s)(13+s)}{(2+s)(4+s)(6+s)(8+s)} B_{5}(8+s) .
\end{aligned}
$$


Inspection of (58) shows that it establishes the hyperclosure of $\Delta_{5}$ (odd) on the basis of the hyperclosure of all $B_{5}$ (odd). To summarize, and say something also about even integer arguments, we hereby state:

Theorem 9 [Partial hyperclosure for $n \geq 5$ ] We have, for any integer $s$,

$2(s-2) s(s+1) B_{5}(s-4)-(s+3)\left\{s(3 s+7) B_{5}(s-2)-(s+4)(s+5) B_{5}(s)\right\}=60 C_{2,0}(s, 3)$,

For $n=6$, a similar superposition-this time involving four B-terms-is

$$
\begin{gathered}
-6 s^{2}(s-2)(s-4) B_{6}(s-6)+(s-2) s(s+2)(18+11 s) B_{6}(s-4)- \\
(3+s)(4+s)\left\{2 s(8+3 s) B_{6}(s-2)-(5+s)(6+s) B_{6}(s)\right\}=360 C_{2,0}(s, 4) .
\end{gathered}
$$

It follows that for any integer s, each of these B-superpositions is hyperclosed; moreover, for every odd integer $s$, each of $B_{5}(s)$ and $\Delta_{5}(s)$ is hyperclosed.

Proof: The two recurrences follow from a combination of $(39,44)$, and Theorem 4 implies the hyperclosure of the two superpositions. Since we have determined the pair $B_{5}( \pm 1)$ at the beginning of this section, the first recurrence in the theorem is thereby ignited, so $B_{5}(s)$ is hyperclosed for all odd $s$. Hyperclosure for $\Delta_{5}$ (odd) follows from (58). QED

Remark: Hence, we can assert that the only potentially problematic values for $n=5$ are $B_{5}(-2 n)$ and $\Delta_{5}(-2 n)$ for positive integer $n$. In this setting both (57) and (58) engage a variety of thornier issues generated by the poles. But even here we discover that all $B_{5}$ (even) can be resolved with the adjunction of $B_{5}(-6)$ (equivalently $\mathcal{K}_{4}$ ) and $B_{5}(-4)$ (equivalently $\mathcal{K}_{5}$ ), and this should yield in turn some, if not all, of the $\Delta_{5}$ (even). We should admit that the $n=6$ recursion is basically all we know about 6 dimensional cases: We do not know a single nontrivial closed form for a $B_{6}(s)$ or a $\Delta_{6}(s)$. We can, however, write

$$
\begin{aligned}
B_{6}(s) & =\frac{8}{(s+2)(s+4)(s+6)} \int_{0}^{\pi / 4} \int_{0}^{\pi / 4} \int_{0}^{\pi / 4}\left(\sec ^{2}(a)+\sec ^{2}(b)+\sec ^{2}(c)\right)^{3+s / 2} d a d b d c \\
& -\frac{\pi}{2} \frac{3}{s+2} B_{4}(s+2)-\frac{\pi^{2}}{4} \frac{3}{(s+2)(s+4)} B_{2}(s+4)
\end{aligned}
$$

valid for all values of $s$. In particular

$$
\begin{aligned}
B_{6}(-7)= & -\frac{8}{15} \int_{0}^{\pi / 4} \int_{0}^{\pi / 4} \int_{0}^{\pi / 4} \frac{d a d b d c}{\sqrt{\sec ^{2}(a)+\sec ^{2}(b)+\sec ^{2}(c)}} \\
& +\frac{3 \sqrt{2}}{5} \arctan \left(\frac{1}{\sqrt{8}}\right) \pi+\frac{3 \sqrt{2}}{32} \pi^{2} .
\end{aligned}
$$


Moreover, the triple integral above resolves to

$$
\begin{aligned}
& \frac{1}{2} \int_{0}^{\pi / 4} \int_{0}^{\pi / 4} \frac{\operatorname{arcsec}\left(1+\sec ^{2}(a)+\sec ^{2}(b)\right)}{\sqrt{\sec ^{2}(a)+\sec ^{2}(b)}} d a d b=-\frac{3}{4} \int_{1}^{2} \frac{\operatorname{arcsec}(z+3) \operatorname{arcsec}(z)}{(z+2)^{3 / 2} \sqrt{z+1}} d z \\
+ & \frac{\pi}{\sqrt{8}}\left\{2 \arctan \left(\frac{3}{4}\right)-3 \arctan \left(\frac{7}{8} \sqrt{2}\right)+\sqrt{\frac{3}{2}} \arctan (2 \sqrt{6})\right\} .
\end{aligned}
$$

Thus we arrive at

$B_{6}(-7)=\frac{2}{5} \mathcal{K}_{8}+\frac{7 \sqrt{2}}{60} \pi^{2}-\frac{7 \sqrt{2}}{5} \pi \arctan \left(\frac{1}{\sqrt{8}}\right)+\frac{8 \sqrt{2}}{15} \pi \arctan \left(\frac{1}{2}\right)-\frac{2 \sqrt{3}}{15} \pi \arctan (\sqrt{24})$

where an integral form of $\mathcal{K}_{8}$ is exhibited in the next section.

\section{$9 \mathcal{K}$-integrals}

In a set of marvelous preprints, G. Lamb [17] managed to largely resolve "dangling integrals" from our previous work [5]. We called these $\mathcal{K}_{m}$, for $m=0,1,2,3$. Now, via the combination of proof via alternative integration (as with result (71) below), together with the powerful Lamb techniques, we know closed forms such as

$$
\mathcal{K}_{0}:=\int_{0}^{1} \frac{\operatorname{arctanh}\left(\frac{1}{\sqrt{3+y^{2}}}\right)}{1+y^{2}} d y=\frac{3}{2} \operatorname{Ti}_{2}(3-2 \sqrt{2})+\frac{\pi}{4} \log (1+\sqrt{2})-\frac{1}{2} G .
$$

Thus $\mathcal{K}_{0}$ is hyperclosed.

The next integral is perhaps the most difficult one of the present treatment. Along the way to the following answer-having started with the Lamb method - were stages of symbolics, at times involving over 28000 characters.

$$
\begin{aligned}
\mathcal{K}_{1}:= & \int_{3}^{4} \frac{\operatorname{arcsec}(x)}{\sqrt{x^{2}-4 x+3}} d x \\
= & \mathrm{Cl}_{2}(\theta)-\mathrm{Cl}_{2}\left(\theta+\frac{\pi}{3}\right)-\mathrm{Cl}_{2}\left(\theta-\frac{\pi}{2}\right)+\mathrm{Cl}_{2}\left(\theta-\frac{\pi}{6}\right)-\mathrm{Cl}_{2}\left(3 \theta+\frac{\pi}{3}\right) \\
& +\mathrm{Cl}_{2}\left(3 \theta+\frac{2 \pi}{3}\right)-\mathrm{Cl}_{2}\left(3 \theta-\frac{5 \pi}{6}\right)+\mathrm{Cl}_{2}\left(3 \theta+\frac{5 \pi}{6}\right)+\left(6 \theta-\frac{5 \pi}{2}\right) \log (2-\sqrt{3}) .
\end{aligned}
$$

where $\mathrm{Cl}_{2}(\theta):=\sum_{n=1}^{\infty} \sin (n \theta) / n^{2}$ is the Clausen function, and

$$
3 \theta:=\arctan \left(\frac{16-3 \sqrt{15}}{11}\right)+\pi
$$


It may well be that this closed form for $\mathcal{K}_{1}$ can be further simplified.

Not so hard, but certainly nontrivial, is

$$
\begin{aligned}
\mathcal{K}_{2} & :=\int_{0}^{\pi / 4} \sqrt{1+\sec ^{2}(a)} \arctan \left(\frac{1}{\sqrt{1+\sec ^{2}(a)}}\right) d a \\
& =\frac{1}{2} \operatorname{Ti}_{2}(-2+\sqrt{3})+\frac{\pi}{8} \log (2+\sqrt{3})+\frac{\pi^{2}}{32} .
\end{aligned}
$$

Finally, we have resolved a dangling integral from [5] involving dimension $n=5$, as

$$
\begin{aligned}
\mathcal{K}_{3} & :=\int_{0}^{\pi / 4} \int_{0}^{\pi / 4} \sqrt{1+\sec ^{2}(a)+\sec ^{2}(b)} d a d b \\
& =\frac{1}{4} \log (2+\sqrt{3})+\frac{1}{48} \pi^{2}-\frac{1}{10} B_{5}(-3) .
\end{aligned}
$$

Indeed the formula for the jellium constant $J_{5}$ given in $[5, \S 7.3]$ yields precisely $(64)$ and the closed form for $B_{5}(-3)$ is listed in Table 4 .

Moving on, we hereby define additional $\mathcal{K}$-integrals, starting with

$$
\mathcal{K}_{4}:=\int_{0}^{\pi / 4} \frac{\arctan \left(\frac{1}{\sqrt{2+\sec ^{2}(t)}}\right)}{\sqrt{2+\sec ^{2}(t)}} d t
$$

and

$$
\mathcal{K}_{5}:=\int_{0}^{\pi / 4} \int_{0}^{\pi / 4} \log \left(1+\sec ^{2}(a)+\sec ^{2}(b)\right) d a d b
$$

Following suggestions in [18], and significant symbolic computation, we can express $\mathcal{K}_{4}$ explicitly in terms of complex dilogarithms. The CAS produced an answer which with some massaging became:

$$
\begin{aligned}
\frac{1}{\sqrt{8}} \mathcal{K}_{4} & =\frac{7 \pi}{16} \arctan (2)+\frac{3 \pi}{16} \arctan (27+20 \sqrt{2})-\frac{13 \pi^{2}}{64} \\
& +\frac{1}{8} \Re\left\{\operatorname{Li}_{2}(\sqrt{2}+i)-\operatorname{Li}_{2}(-\sqrt{2}+i)\right\} \\
& +\frac{1}{4} \Re\left\{\operatorname{Li}_{2}\left(\frac{4}{5}-\frac{3}{5} i-\frac{3}{5} \sqrt{2}-\frac{4}{5} i \sqrt{2}\right)-\operatorname{Li}_{2}\left(\frac{4}{5}-\frac{3}{5} i+\frac{3}{5} \sqrt{2}+\frac{4}{5} i \sqrt{2}\right)\right\} \\
& +\frac{1}{8} \Re\left\{\operatorname{Li}_{2}\left(\frac{4}{15}-\frac{1}{5} i-\frac{1}{5} \sqrt{2}-\frac{4}{15} i \sqrt{2}\right)-\operatorname{Li}_{2}\left(\frac{4}{15}-\frac{1}{5} i+\frac{1}{5} \sqrt{2}+\frac{4}{15} i \sqrt{2}\right)\right\} .
\end{aligned}
$$


The arguments of the dilogarithms here solve an interesting, degree-12 polynomial system. One may use dilogarithm reflection formulae to obtain closed forms such as

$$
\begin{aligned}
\sqrt{8} \mathcal{K}_{4}= & -\operatorname{Li}_{2}\left(\frac{1}{\sqrt{3}}, \theta\right)+\operatorname{Li}_{2}\left(\frac{1}{\sqrt{3}}, \pi-\theta\right)-\operatorname{Li}_{2}\left(\frac{1}{\sqrt{3}}, \pi-\alpha\right)+\operatorname{Li}_{2}\left(\frac{1}{\sqrt{3}}, \beta\right) \\
& +(\pi-8 \theta) \arctan \left(\frac{1}{2}\right),
\end{aligned}
$$

where

$$
\theta:=\arctan \left(\frac{1}{\sqrt{2}}\right), \quad \alpha:=\arctan \left(\frac{3+4 \sqrt{2}}{-4+3 \sqrt{2}}\right) \quad \text { and } \quad \beta:=\arctan \left(\frac{-3+4 \sqrt{2}}{4+3 \sqrt{2}}\right),
$$

with the Lewin generalization $\operatorname{Li}_{2}(r, \phi):=\Re \mathrm{Li}_{2}\left(r e^{i \phi}\right)$; see [19, A2.5 (1)]. It is immediate that $\mathcal{K}_{4}$ is hyperclosed, since in our $\mathcal{K}_{4}$ formulae any arguments $r e^{ \pm i \theta}$ are all algebraic, and $2 \Re \mathrm{Li}_{2}(z)=\mathrm{Li}_{2}(z)+\mathrm{Li}_{2}\left(z^{*}\right)$.

So, we know that $\mathcal{K}_{0,1,2,3,4}$ are all hyperclosed but we do not know this for $\mathcal{K}_{5}$. Various recondite, but alas partial results can be derived, starting typically from (66) and employing coordinates $\tan a, \tan b$ over the unit 2-square. One can obtain such as a 1dimensional-integral form

$$
\begin{aligned}
\mathcal{K}_{5} & =\int_{0}^{1} \frac{\arctan (\sqrt{\mathrm{t}}) \arctan \left(\frac{1}{\sqrt{3+\mathrm{t}}}\right)}{\sqrt{3+t}(2+t)} d t-\frac{\pi}{2} G+\frac{\pi^{2}}{16} \ln (3) \\
& +\frac{\pi}{2}(3-\sqrt{8}) \mathrm{Ti}_{2}(3-\sqrt{8})+\frac{\pi}{12} \operatorname{Ti}_{2}\left(\frac{1}{3}\right)+\frac{\pi^{2}}{8} \ln (\sqrt{2}-1),
\end{aligned}
$$

or an efficient sum

$$
\mathcal{K}_{5}=\frac{\pi^{2}}{16} \log 3-\sum_{m \geq 1} \frac{1}{m 3^{m}} \sum_{j=0}^{m}\left(\begin{array}{c}
m \\
j
\end{array}\right)\left(h_{j}-\frac{\pi}{4}\right)\left(h_{m-j}-\frac{\pi}{4}\right),
$$

where $h_{k}:=1-1 / 3+1 / 5-\cdots \pm 1 /(2 j-1)$, with $h_{0}:=0$. This summatory representation allows extreme-precision evaluation of $\mathcal{K}_{5}$ without recourse to quadrature per se.

Now to the concept of alternative integration: It is highly interesting that the boxintegral theory can sometimes be used to resolve previously unknown integrals that have, on the face of it, little to do with $n$-dimensional boxes. A canonical example is the case of $\Delta_{3}(-2)$, which is obtained through the rather delicate limit process $s \rightarrow-2$ in (56) in order to complete hyperclosure for $n=3$. But when we attempted to evaluate $\Delta_{3}(-2)$ from the alternative formula

$$
\Delta_{3}(-2)=8 \int_{\vec{r} \in[0,1]^{3}} \frac{(1-x)(1-y)(1-z)}{x^{2}+y^{2}+z^{2}} d x d y d z
$$


we ended up with a single, troublesome arctan integral as the "dangling term." But the $(s \rightarrow-2)$ limit procedure does a complete hypergeometric breakdown, and all of this proves the peculiar result

$$
\begin{aligned}
\mathcal{K}_{6} & :=\int_{0}^{1} \sqrt{1+z^{2}} \arctan \left(\frac{1}{\sqrt{1+z^{2}}}\right) d z \\
& =\frac{1}{2}-\frac{G}{2}+\frac{\pi}{4} \log (1+\sqrt{2})+\frac{1}{2} \operatorname{Ti}_{2}(3-2 \sqrt{2}) .
\end{aligned}
$$

We do not give here a direct proof of this integral relation, although G. Lamb has now provided a fine analytic evaluation of $\mathcal{K}_{6}$; see [18]. Another example of an indirect integral resolution is

$$
\begin{aligned}
\mathcal{K}_{7} & :=\int_{0}^{\pi / 4} \log \left(1+\sqrt{2+\sec ^{2} t}\right) d t \\
& =-G+2 \mathrm{Ti}_{2}(3-2 \sqrt{2})+\frac{\pi}{2} \log (1+\sqrt{2}) .
\end{aligned}
$$

Finally, we define

$$
\mathcal{K}_{8}:=\int_{1}^{2} \frac{\operatorname{arcsec}(x+3) \operatorname{arcsec}(x)}{\sqrt{(x+1)(x+2)^{3}}} d x,
$$

and observe that $\mathcal{K}_{8}$, along with the elusive $\mathcal{K}_{5}$, are the only unresolved instances of $\mathcal{K}_{0,1, \ldots, 8}$

Again, knowledge of $\mathcal{K}_{8}$ would give us $B_{6}(-7)$, see $(73)$, while hyperclosure of $\mathcal{K}_{5}$ would establish hyperclosure of all $B_{5}$ (integer) and thereby probably of all $\Delta_{5}$ (integer).

\section{Curiosities}

One new result of some interest is that another unphysical, but valid analytically continued value discussed in our previous work [5], namely

$$
B_{4}(-5)=-0.961203932689953457121659780024745 \ldots
$$

is now known in closed form. In fact, this previously mysterious analytic-continuation value is $-\sqrt{8} \arctan (1 / \sqrt{8})$ (see Tables and the last part of Theorem 5). However, there are still many unexplained empirical phenomena. For example we do not know in closed form the zero of $\Delta_{2}(s)$ at

$$
s=-5.1378771851212623537 \ldots,
$$

though we have developed herein enough machinery to find this zero to extreme precision. (See Figure 1 for a pictorial of the zero.) Moreover, we are not aware of any other zeros in 
$n=2$ or any other dimension; evidently, this zero of $\Delta_{2}$ is some kind of anomaly. There is also an empirical local minimum for $B_{2}$, at

$$
s=3.6675667756027541433 \ldots,
$$

and again a closed form is unknown.

Another curiosity is that some statistical quantities over the hypercube are almost trivial to resolve. For example, the exponential expectations

$$
\begin{gathered}
E_{n}(\lambda):=\left\langle e^{-\lambda^{2}|\vec{r}|^{2}}\right\rangle_{\vec{r} \in[0,1]^{n}}, \\
F_{n}(\lambda):=\left\langle e^{-\lambda^{2}|\vec{r}-\vec{q}|^{2}}\right\rangle_{\vec{r}, \vec{q} \in[0,1]^{n}}
\end{gathered}
$$

for constant $\lambda$ do not need to be obtained from a generating function or series or anything complicated. Instead, closed forms are immediate, based on the defining relations $(3,4)$ :

$$
\begin{gathered}
E_{n}(\lambda)=b^{n}(\lambda)=\left(\frac{\pi}{2 \lambda}\right)^{n} \operatorname{erf}^{n}(\lambda) \\
F_{n}(\lambda)=d^{n}(\lambda)
\end{gathered}
$$

The allure of such closed forms is that they hold in all dimensions, and may well replace the box integrals $B_{n}(s), \Delta_{n}(s)$ as the right tools to assess the statistical character of point clouds, again as in reference [14]. In spite of this streamlined approach to cloud measures, it seems (heuristically) that both of

$$
\left\langle e^{-\kappa|\vec{r}|}\right\rangle_{\vec{r} \in[0,1]^{n}}, \quad\left\langle e^{-\kappa|\vec{r}-\vec{q}|}\right\rangle_{\vec{r}, \vec{q} \in[0,1]^{n}}
$$

should be extremely difficult to evaluate in any general way. Even if one were to revert to series expansion of the exponential, one would need $B_{n}(s)$ for all nonnegative integers $s$, yet we have met with extreme difficulties when $n>5$. This is not to claim some clever prescription - a novel use of recurrences, or differential relations - would not solve this problem.

And what about noninteger arguments for the box integrals? One might extend the definition of hypergeometric closure based on such findings as

$$
B_{3}\left(-\frac{7}{2}\right)=-2 \pi-\frac{8}{5 \cdot 2^{\frac{1}{4}}} F_{1}\left(\frac{5}{4} ; \frac{1}{2}, \frac{3}{4} ; \frac{9}{4} ; \frac{1}{2},-\frac{1}{2}\right)+\frac{16}{5} \sqrt{\pi} \frac{\Gamma\left(\frac{9}{4}\right)}{\Gamma\left(\frac{7}{4}\right)}{ }_{2} F_{1}\left(\frac{5}{4}, \frac{3}{4} ; \frac{7}{4} ;-1\right) .
$$

Here, $F_{1}$ is the Appell hypergeometric function - defined as a series in two variables [2]. Thus we expect entities such as $B_{3}$ (odd/2) to involve such as the Appell hypergeometric. Such examples suggest that there might be a kind of higher level of hypergeometric-closure theory for noninteger rational arguments $s$. 


\section{Conjectures and open questions}

We conclude by reiterating the following open questions.

1. Is it a reasonable conjecture that every box integral $B_{\text {integer }}$ (integer), $\Delta_{\text {integer }}$ (integer) be hyperclosed? That is, could it be that our results for $n \leq 4$ actually are extensible for all positive integer $n$ ?

2. Is there a general evaluation (hyperclosed or not) of $B_{n}(-n-1)$ for all natural numbers $n$ ?

3. What transpires for noninteger but rational $s$ in dimension $n \leq 5$ (see relation (74)?

4. What transpires for dimension $n>5$, say just for any of $B_{6}(s) ; s \in(1,-1,-2,-4)$ ?

5. Referring to Figure 1, what is a closed form for the mysterious zero? Are there any other (possibly complex) zeros of any $\Delta_{n}$ whatsoever? Is there a closed form for the local minimum of $B_{2}(s)$ at $s \approx 3.667 \ldots$ ?

Acknowledgments. The authors are grateful to T. Wieting for clarifications on hypergeometric algebra, as well as to G. Lamb, J. Philip, M. Trott, and E. Weisstein for their contributions to box-integral theory. In particular, the Clausen terms in earlier formulae for $\Delta_{4}(1), \Delta_{5}(1)$ in [5] needed repair; we thank J. Philip for pointing this out in his delightful paper [21]. ${ }^{9}$

\section{References}

[1] R. Anderssen, R. Brent, D. Daley, and P. Moran, "Concerning $\int_{0}^{1} \cdots \int_{0}^{1}\left(x_{1}^{2}+\cdots x_{n}^{2}\right)^{\frac{1}{2}} d x_{1} \cdots d x_{n}$ and a Taylor series method," SIAM J. Applied Math., 30 (1976), 22-30.

[2] M. Abramowitz and I.A. Stegun, Handbook of Mathematical Functions, NBS (now NIST), 1965. See also http://dlmf.nist.gov/.

[3] David H. Bailey and Jonathan M. Borwein, "Highly parallel, high-precision numerical integration," Int. Journal of Computational Science and Engineering, accepted Jan. 2008.

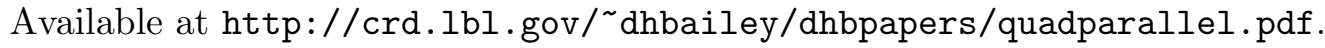

\footnotetext{
${ }^{9}$ Therein his formula for $\Delta_{4}(1)$ has yet a newer misprint. The present authors believe that the best way to ascertain what version of a recondite closed form be the correct one - across publications, say - is to test expressions via extreme numerical precision.
} 
[4] David H. Bailey and Jonathan M. Borwein, "Effective error bounds for Euler-Maclaurin-based quadrature schemes," 20th Annual HPCS Proceedings, 7 pp., IEEE CD, May 2006. Available at http://crd.lbl.gov/ dhbailey/dhbpapers/em-error.pdf.

[5] D.H. Bailey, J.M. Borwein and R.E. Crandall, "Box integrals," Journal of Computational and Applied Mathematics, 206 (2007), 196-208. [D-drive Preprint 320].

[6] D.H. Bailey, J.M. Borwein and R.E. Crandall, "Integrals of the Ising class," J. Phys. A., 39 (2006), 12271-12302. Available at http://crd.lbl.gov/ dhbailey/dhbpapers/Ising.pdf.

[7] David H. Bailey, Jonathan M. Borwein, Vishaal Kapoor, and Eric W. Weisstein, "Ten problems in experimental mathematics," Amer. Mathematical Monthly, 113 (2006), 481-509. Available at http://locutus.cs.dal.ca:8088/archive/00000316/

[8] David H. Bailey and David J. Broadhurst, "Parallel integer relation detection: Techniques and applications," Mathematics of Computation, 70 (Oct 2000), 1719-1736.

[9] David H. Bailey, Xiaoye S. Li and Karthik Jeyabalan, "A comparison of three high-precision quadrature schemes," Experimental Mathematics, 14 (2005), 317-329. Available at http://crd.lbl.gov/ dhbailey/dhbpapers/quadrature.pdf.

[10] David H. Bailey, Yozo Hida, Xiaoye S. Li and Brandon Thompson, "ARPREC: An arbitrary precision computation package," Sept 2002. Available at http://crd.lbl.gov/ dhbailey/dhbpapers/arprec.pdf.

[11] Jonathan M. Borwein and David H. Bailey, Mathematics by Experiment, AK Peters, 2003. Second edition, 2008. See also http://www. experimentalmath.info.

[12] J. M. Borwein and B. Salvy, "A proof of a recursion for Bessel moments," Exp. Mathematics, vol. 17 (2008), 223-230.

[13] Chaunming Zong, The Cube-A Window to Convex and Discrete Geometry, Cambridge Tracts in Mathematics, 2006.

[14] R.E. Crandall, C. Joe, and T. Mehoke, "On the fractal distribution of brain synapses," to appear, 2009.

[15] Helaman R. P. Ferguson, David H. Bailey and Stephen Arno, "Analysis of PSLQ, an integer relation finding algorithm," Mathematics of Computation, 68 (Jan 1999), 351-369.

[16] Wolfram Koepf, Hypergeometric Summation: An Algorithmic Approach to Summation and Special Function Identities, American Mathematical Society, Providence, RI, 1998.

[17] G. Lamb, "An evaluation of the integral $\mathcal{K}_{0}$," "A method of evaluating the integral $\mathcal{K}_{1}$," and "An approach to evaluating the integral $\mathcal{K}_{3}$," preprint collection, 2006. 
[18] G. Lamb, "An evaluation of the integral $\mathcal{K}_{6}$," and "An approach to evaluating the integral $\mathcal{K}_{4}$," preprint collection, 2009.

[19] Leonard Lewin, Polylogarithms and Associated Functions, North Holland, 1981.

[20] Jack Dongarra, Jim Bunch, Cleve Moler, Pete Stewart, LINPACK User's Guide, SIAM, Philadelphia, 1979.

[21] J. Philip, "The distance between two random points in a 4- and 5-cube," preprint, 2008.

[22] D. Robbins, "Average distance between two points in a box," Amer. Mathematical Monthly, 85 (1978), 278.

[23] Charles Schwartz, "Numerical integration of analytic functions," Journal of Computational Physics, 4 (1969), 19-29.

[24] Hidetosi Takahasi and Masatake Mori, "Quadrature formulas obtained by variable transformation," Numerische Mathematik, 21 (1973), 206-219.

[25] M. Trott, Private communication, 2005.

[26] M. Trott, "The area of a random triangle," Mathematica Journal, 7 (1998), 189-198.

[27] E. Weisstein, "Hypercube line picking," available at http://mathworld. wolfram.com/HypercubeLinePicking.html. 


\section{Appendix: Compendium of proven closed forms}

Numerical tables in support of the following closed forms are to be found at http://crd.lbl.gov/ dhbailey/dhbpapers/box-int-b-delta.txt 


\begin{tabular}{|c|c|c|}
\hline$n$ & $s$ & $B_{n}(s)$ \\
\hline any & even $\geq 0$ & rational: $B_{2}(2)=2 / 3$ \\
1 & -1 & $\infty$ \\
1 & any & $\frac{1}{s+1}$ \\
\hline 2 & -4 & $-\frac{1}{4}-\frac{\pi}{8}$ \\
2 & -3 & $-\sqrt{2}$ \\
2 & -2 & $\infty$ \\
2 & -1 & $2 \log (1+\sqrt{2})$ \\
2 & 1 & $\frac{1}{3} \sqrt{2}+\frac{1}{3} \log (1+\sqrt{2})$ \\
2 & 3 & $\frac{7}{5} \sqrt{2}+\frac{3}{20} \log (1+\sqrt{2})$ \\
2 & any & $\frac{2}{2+s}{ }_{2} F_{1}\left(\frac{1}{2},-\frac{s}{2} ; \frac{3}{2} ;-1\right)$ \\
\hline
\end{tabular}

Table 1: Some relatively easy evaluations for $B_{n}(s)$. Every $B_{k}$ (integer) for $k=1,2$ can be given a closed form (see Theorem 6). 


\begin{tabular}{|c|c|c|}
\hline$n$ & $s$ & $B_{n}(s)$ \\
\hline 3 & -5 & $-\frac{1}{6} \sqrt{3}-\frac{1}{12} \pi$ \\
3 & -4 & $-\frac{3}{2} \sqrt{2} \arctan \frac{1}{\sqrt{2}}$ \\
3 & -3 & $\infty$ \\
3 & -2 & $-3 G+\frac{3}{2} \pi \log (1+\sqrt{2})+3 \mathrm{Ti}_{2}(3-2 \sqrt{2})$ \\
3 & -1 & $-\frac{1}{4} \pi+\frac{3}{2} \log (2+\sqrt{3})$ \\
3 & 1 & $\frac{1}{4} \sqrt{3}-\frac{1}{24} \pi+\frac{1}{2} \log (2+\sqrt{3})$ \\
3 & 3 & $\frac{2}{5} \sqrt{3}-\frac{1}{60} \pi-\frac{7}{20} \log (2+\sqrt{3})$ \\
3 & any & Integral representation $(25)$, relation (38) \\
\hline
\end{tabular}

Table 2: Example evaluations of $B_{3}(s)$. Every $B_{3}$ (integer) can be given a closed form, being as we can express every $B_{3}$ in terms of a function $C_{2,0}$ which we have shown to be hyperclosed at relevant arguments. 


\begin{tabular}{|c|c|c|}
\hline$n$ & $s$ & $B_{n}(s)$ \\
\hline 4 & -5 & $-\sqrt{8} \arctan \left(\frac{1}{\sqrt{8}}\right)$ \\
4 & -4 & $\infty$ \\
4 & -3 & $4 G-12 \mathrm{Ti}_{2}(3-2 \sqrt{2})$ \\
4 & -2 & $\pi \log (2+\sqrt{3})-2 G-\frac{\pi^{2}}{8}$ \\
4 & -1 & $2 \log 3-\frac{2}{3} G+2 \mathrm{Ti}_{2}(3-2 \sqrt{2})-\sqrt{8} \arctan \left(\frac{1}{\sqrt{8}}\right)$ \\
4 & 1 & $\frac{2}{5}-\frac{G}{10}+\frac{3}{10} \mathrm{Ti}_{2}(3-2 \sqrt{2})+\log 3-\frac{7 \sqrt{2}}{10} \arctan \left(\frac{1}{\sqrt{8}}\right)$ \\
4 & any & Integral representation $(27)$, recursions $(44)$ \\
\hline
\end{tabular}

Table 3: Example evaluations of $B_{4}(s)$. Every $B_{4}$ (integer) can be given a closed form, on the basis of recursions and "ignition values" such as the pair $B_{4}(-1), B_{4}(-2)$. 


\begin{tabular}{|c|c|c|}
\hline$n$ & $s$ & $B_{n}(s)$ \\
\hline 5 & -8 & $\frac{25}{36} \mathcal{K}_{4}-\frac{25}{288} \pi \sqrt{2} \arctan \left(\frac{1}{\sqrt{2}}\right)-\frac{5}{72} \arctan \left(\frac{1}{2}\right)$ \\
\hline 5 & -6 & $5 \mathcal{K}_{4}-\frac{5}{8} \pi \sqrt{2} \arctan \left(\frac{1}{\sqrt{2}}\right)$ \\
\hline 5 & -5 & $\infty$ \\
\hline 5 & -4 & $-5 \mathcal{K}_{5}-\frac{5}{2} \pi G+\frac{5}{4} \pi^{2} \log (1+\sqrt{2})+\frac{5}{2} \pi \mathrm{Ti}_{2}(3-2 \sqrt{2})$ \\
\hline 5 & -3 & $\begin{array}{l}-\frac{110}{9} G-10 \log (2-\sqrt{3}) \theta-\frac{1}{8} \pi^{2}+5 \log \left(\frac{1+\sqrt{5}}{2}\right)-\frac{5}{2} \sqrt{3} \arctan \left(\frac{1}{\sqrt{15}}\right) \\
-10 \mathrm{Cl}_{2}\left(\frac{1}{3} \theta+\frac{1}{3} \pi\right)+10 \mathrm{Cl}_{2}\left(\frac{1}{3} \theta-\frac{1}{6} \pi\right) \\
+\frac{10}{3} \mathrm{Cl}_{2}\left(\theta+\frac{1}{6} \pi\right)+\frac{20}{3} \mathrm{Cl}_{2}\left(\theta+\frac{4}{3} \pi\right)-\frac{10}{3} \mathrm{Cl}_{2}\left(\theta+\frac{5}{3} \pi\right)-\frac{20}{3} \mathrm{Cl}_{2}\left(\theta+\frac{11}{6} \pi\right)\end{array}$ \\
\hline 5 & -2 & $\frac{8}{3} B_{5}(-6)-\frac{1}{3} B_{5}(-4)+\frac{5}{2} \pi \log (3)+10 \mathrm{Ti}_{2}\left(\frac{1}{3}\right)-10 G$ \\
\hline 5 & -1 & $\begin{array}{c}-\frac{110}{27} G+\frac{10}{3} \log (2-\sqrt{3}) \theta+\frac{1}{48} \pi^{2}+5 \log \left(\frac{1+\sqrt{5}}{2}\right)-\frac{5}{2} \sqrt{3} \arctan \left(\frac{1}{\sqrt{15}}\right) \\
+\frac{10}{3} \mathrm{Cl}_{2}\left(\frac{1}{3} \theta+\frac{1}{3} \pi\right)-\frac{10}{3} \mathrm{Cl}_{2}\left(\frac{1}{3} \theta-\frac{1}{6} \pi\right) \\
-\frac{10}{9} \mathrm{Cl}_{2}\left(\theta+\frac{1}{6} \pi\right)+\frac{20}{3} \mathrm{Cl}_{2}\left(\theta+\frac{4}{3} \pi\right)-\frac{10}{3} \mathrm{Cl}_{2}\left(\theta+\frac{5}{3} \pi\right)+\frac{20}{9} \mathrm{Cl}_{2}\left(\theta+\frac{11}{6} \pi\right)\end{array}$ \\
\hline 5 & 1 & $\begin{array}{c}-\frac{77}{81} G+\frac{7}{9} \log (2-\sqrt{3}) \theta+\frac{1}{360} \pi^{2}+\frac{1}{6} \sqrt{5}+\frac{10}{3} \log \left(\frac{1+\sqrt{5}}{2}\right)-\frac{4}{3} \sqrt{3} \arctan \left(\frac{1}{\sqrt{15}}\right)+ \\
\frac{7}{9} \mathrm{Cl}_{2}\left(\frac{1}{3} \theta+\frac{1}{3} \pi\right)-\frac{7}{9} \mathrm{Cl}_{2}\left(\frac{1}{3} \theta-\frac{1}{6} \pi\right) \\
-\frac{7}{27} \mathrm{Cl}_{2}\left(\theta+\frac{1}{6} \pi\right)-\frac{14}{27} \mathrm{Cl}_{2}\left(\theta+\frac{4}{3} \pi\right)+\frac{7}{27} \mathrm{Cl}_{2}\left(\theta+\frac{5}{3} \pi\right)+\frac{14}{27} \mathrm{Cl}_{2}\left(\theta+\frac{11}{6} \pi\right)\end{array}$ \\
\hline 5 & any & $(57)$, recursions $(44)$ \\
\hline
\end{tabular}

Table 4: Example evaluations of $B_{5}(s)$. Here $\theta=\arctan \left(\frac{16-3 \sqrt{15}}{11}\right)$. We have proven that every $B_{5}$ (odd) is hyperclosed. Note that $\mathcal{K}_{4}$ here is known, hyperclosed; yet, an unresolved ("dangling") integral $K_{5}$ is not yet known to be hyperclosed, but may well be. Resolution of $\mathcal{K}_{5}$ would serve to establish all the $B_{5}$ (even) by settling $B_{5}(-2), B_{5}(-4)$, and likely lead thus to resolution of all the $\Delta_{5}$ (integer). 


\begin{tabular}{|c|c|c|}
\hline$n$ & $s$ & $\Delta_{n}(s)$ \\
\hline any & even $\geq 0$ & rational: $\Delta_{2}(2)=1 / 3$ \\
1 & $-1,-2$ & $\frac{2}{s+1}-\frac{2}{s+2}$ \\
1 & any & $\frac{4}{3}+\frac{8}{9} \sqrt{2}$ \\
2 & -5 & $\infty$ \\
2 & $-2,-3,-4$ & $\frac{4}{3}-\frac{4}{3} \sqrt{2}+4 \log (1+\sqrt{2})$ \\
2 & -1 & $\frac{2}{15}+\frac{1}{15} \sqrt{2}+\frac{1}{3} \log (1+\sqrt{2})$ \\
2 & 1 & Formula $(55)$ \\
2 & any & \\
\hline
\end{tabular}

Table 5: Some relatively easy evaluations for $\Delta_{n}(s)$. Every $\Delta_{k}$ (integer) for $k=1,2$ can be given a closed form (see Theorem 8). (Note: The closed form for $\Delta_{2}(-1)$ here has been repaired w.r.t. the previous work [5].) 


\begin{tabular}{|c|c|c|}
\hline$n$ & $s$ & $\Delta_{n}(s)$ \\
\hline 3 & -7 & $\frac{4}{5}-\frac{16 \sqrt{2}}{15}+\frac{2 \sqrt{3}}{5}+\frac{\pi}{15}$ \\
3 & $-3,-4,-5,-6$ & $\infty$ \\
3 & -2 & $2 \pi-12 G+12 \operatorname{Ti}_{2}(3-2 \sqrt{2})+6 \pi \log (1+\sqrt{2})+2 \log 2-\frac{5}{2} \log 3-8 \sqrt{2} \arctan \left(\frac{1}{\sqrt{2}}\right)$ \\
3 & -1 & $\frac{2}{5}-\frac{2}{3} \pi+\frac{2}{5} \sqrt{2}-\frac{4}{5} \sqrt{3}+2 \log (1+\sqrt{2})+12 \log \left(\frac{1+\sqrt{3}}{\sqrt{2}}\right)-4 \log (2+\sqrt{3})$ \\
3 & 1 & $-\frac{118}{21}-\frac{2}{3} \pi+\frac{34}{21} \sqrt{2}-\frac{4}{7} \sqrt{3}+2 \log (1+\sqrt{2})+8 \log \left(\frac{1+\sqrt{3}}{\sqrt{2}}\right)$ \\
3 & 3 & $-\frac{1}{105}-\frac{2}{105} \pi+\frac{73}{840} \sqrt{2}+\frac{1}{35} \sqrt{3}+\frac{3}{56} \log (1+\sqrt{2})+\frac{13}{35} \log \left(\frac{1+\sqrt{3}}{\sqrt{2}}\right)$ \\
3 & any & Formula $(56)$ \\
\hline
\end{tabular}

Table 6: Example evaluations for $\Delta_{3}(s)$. Every $\Delta_{3}$ (integer) can be given a closed form (see Theorem 8). 


\begin{tabular}{|c|c|c|}
\hline$n$ & $s$ & $\Delta_{n}(s)$ \\
\hline 4 & -10 & $-\frac{8}{45} \pi \sqrt{3}-\frac{20}{9} \pi \log (2)+\frac{4}{9} \pi^{2}+\frac{4}{3} \log (2)+\frac{1}{15} \log (3)+\frac{8}{3} \mathrm{Ti}_{2}(3-2 \sqrt{2})$ \\
\hline 4 & -9 & $\begin{array}{c}\frac{16}{5} \pi \sqrt{3}-\frac{32}{3} \pi \log (2)-\frac{2}{3} \pi^{2}+\frac{16}{5} \pi+8 \sqrt{2} \arctan (2 \sqrt{2})-24 \log (2)+\frac{2}{5} \log (3) \\
+12 \pi \log (\sqrt{2}-1)-64 \operatorname{Ti}_{2}(3-2 \sqrt{2})+\frac{160}{3} G\end{array}$ \\
\hline 4 & $-4, \ldots,-8$ & $\infty$ \\
\hline 4 & -3 & $\begin{array}{l}-\frac{128}{15}+\frac{1}{63} \pi-8 \log (1+\sqrt{2})-32 \log (1+\sqrt{3})+16 \log (2)+20 \log (3) \\
\quad-\frac{8}{5} \sqrt{2}+\frac{32}{5} \sqrt{3}-32 \sqrt{2} \arctan \left(\frac{1}{\sqrt{8}}\right)-96 \mathrm{Ti}_{2}(3-2 \sqrt{2})+32 G\end{array}$ \\
\hline 4 & -2 & $\begin{array}{l}-\frac{16}{15} \pi \sqrt{3}+\frac{16}{3} \pi \log (1+\sqrt{3})-\frac{8}{3} \pi \log (2)+4 \pi \log (\sqrt{2}+1)-\frac{2}{3} \pi^{2}+\frac{4}{5} \pi \\
\quad+\frac{8}{5} \sqrt{2} \arctan (2 \sqrt{2})+\frac{2}{5} \log (3)+8 \mathrm{Ti}_{2}(3-2 \sqrt{2})-\frac{40}{3} G\end{array}$ \\
\hline 4 & -1 & $\begin{array}{c}\frac{704}{195}-\frac{8}{39} \pi-\frac{100}{13} \log (3)+\frac{120}{13} \log (2)-\frac{8}{65} \sqrt{2}+\frac{128}{65} \sqrt{3} \\
-\frac{140}{13} \log (1+\sqrt{2})-\frac{32}{13} \log (1+\sqrt{3})+\frac{160}{13} \sqrt{2} \arctan \left(\frac{1}{\sqrt{8}}\right)+\frac{48}{13} G\end{array}$ \\
\hline 4 & 1 & $\begin{array}{c}-\frac{23}{135}-\frac{16}{315} \pi-\frac{52}{105} \log (2)+\frac{197}{420} \log (3)+\frac{73}{630} \sqrt{2}+\frac{8}{105} \sqrt{3} \\
+\frac{1}{14} \log (1+\sqrt{2})+\frac{104}{105} \log (1+\sqrt{3})-\frac{68}{105} \sqrt{2} \arctan \left(\frac{1}{\sqrt{8}}\right)-\frac{4}{15} G+\frac{4}{5} \operatorname{Ti}_{2}(3-2 \sqrt{2})\end{array}$ \\
\hline 4 & any & Formula (56) \\
\hline
\end{tabular}

Table 7: Example evaluations for $\Delta_{4}(s)$. Every $\Delta_{4}$ (integer) can be given a closed form (see Theorem 8) . 


\begin{tabular}{|c|c|c|}
\hline$n$ & $s$ & $\Delta_{n}(s)$ \\
\hline 5 & -12 & $\begin{array}{c}-\frac{2}{3} B_{2}(-6)+\frac{1}{3} B_{2}(-4)+4 B_{3}(-8)-3 B_{3}(-6)+\frac{1}{6} B_{3}(-4)-16 B_{4}(-10)+ \\
\frac{44}{3} B_{4}(-8)-\frac{5}{3} B_{4}(-6)+32 B_{5}(-12)-\frac{100}{3} B_{5}(-10)+\frac{129}{20} B_{5}(-8)-\frac{13}{90} B_{5}(-6)+ \\
\frac{\pi}{108} \sqrt{3}-\frac{7}{800}\end{array}$ \\
\hline 5 & $-5, \ldots,-10$ & $\infty$ \\
\hline 5 & -3 & $\begin{array}{c}-\frac{12304}{63}-\frac{512}{21} \sqrt{2}+\frac{576}{7} \sqrt{3}+\frac{800}{21} \sqrt{5}-\frac{320}{3} B_{2}(3)+\frac{448}{3} B_{2}(5) \\
-320 B_{3}(1)+960 B_{3}(3)-\frac{1792}{3} B_{3}(5)-160 B_{4}(-1)+\frac{4400}{3} B_{4}(1)-\frac{20720}{9} B_{4}(3) \\
+896 B_{4}(5)+32 B_{5}(-3)+\frac{800}{3} B_{5}(-1)-1488 B_{5}(1)+\frac{14336}{9} B_{5}(3)-448 B_{5}(5)\end{array}$ \\
\hline 5 & -1 & $\begin{array}{c}\frac{16388}{189}+\frac{1024}{189} \sqrt{2}-\frac{192}{7} \sqrt{3}-\frac{4000}{189} \sqrt{5}+\frac{64}{3} B_{2}(5)-\frac{192}{7} B_{2}(7)+\frac{320}{3} B_{3}(3) \\
-256 B_{3}(5)+\frac{960}{7} B_{3}(7)+160 B_{4}(1)-\frac{6160}{9} B_{4}(3)+784 B_{4}(5)-\frac{1760}{7} B_{4}(7) \\
+32 B_{5}(-1)-400 B_{5}(1)+\frac{8192}{9} B_{5}(3)-672 B_{5}(5)+\frac{1056}{7} B_{5}(7)\end{array}$ \\
\hline 5 & 1 & $\begin{array}{c}-\frac{1279}{567} G-\frac{4}{189} \pi+\frac{4}{315} \pi^{2}-\frac{449}{3465}+\frac{3239}{62370} \sqrt{2}+\frac{568}{3465} \sqrt{3}-\frac{380}{6237} \sqrt{5} \\
+\frac{295}{252} \log (3)+\frac{1}{54} \log (1+\sqrt{2})+\frac{20}{63} \log (2+\sqrt{3})+\frac{64}{189} \log \left(\frac{1+\sqrt{5}}{2}\right) \\
-\frac{73}{63} \sqrt{2} \arctan \left(\frac{1}{\sqrt{8}}\right)-\frac{8}{21} \sqrt{3} \arctan \left(\frac{1}{\sqrt{15}}\right)+\frac{104}{63} \log (2-\sqrt{3}) \theta \\
+\frac{5}{7} \mathrm{Ti}_{2}(3-2 \sqrt{2})+\frac{104}{63} \mathrm{Cl}_{2}\left(\frac{1}{3} \theta+\frac{1}{3} \pi\right)-\frac{104}{63} \mathrm{Cl}_{2}\left(\frac{1}{3} \theta-\frac{1}{6} \pi\right) \\
-\frac{104}{189} \mathrm{Cl}_{2}\left(\theta+\frac{1}{6} \pi\right)-\frac{208}{189} \mathrm{Cl}_{2}\left(\theta+\frac{4}{3} \pi\right)+\frac{104}{189} \mathrm{Cl}_{2}\left(\theta+\frac{5}{3} \pi\right)+\frac{208}{189} \mathrm{Cl}_{2}\left(\theta+\frac{11}{6} \pi\right)\end{array}$ \\
\hline 5 & any odd & Theorem 9 \\
\hline
\end{tabular}

Table 8: Example evaluations of $\Delta_{5}(s)$. It is proven that every $\Delta_{5}$ (odd) is hyperclosed. Yet, the situation for $\Delta_{5}$ (even) remains open (see caption to Table 4$) . \Delta_{5}$ (even) can be linked to $B$ values, as with the evaluation of $\Delta_{5}(-12)$ here - with the $(12+s) B_{4}(8+s)$ term of relation (58) requiring a residue calculation at the $B_{4}$-pole. 


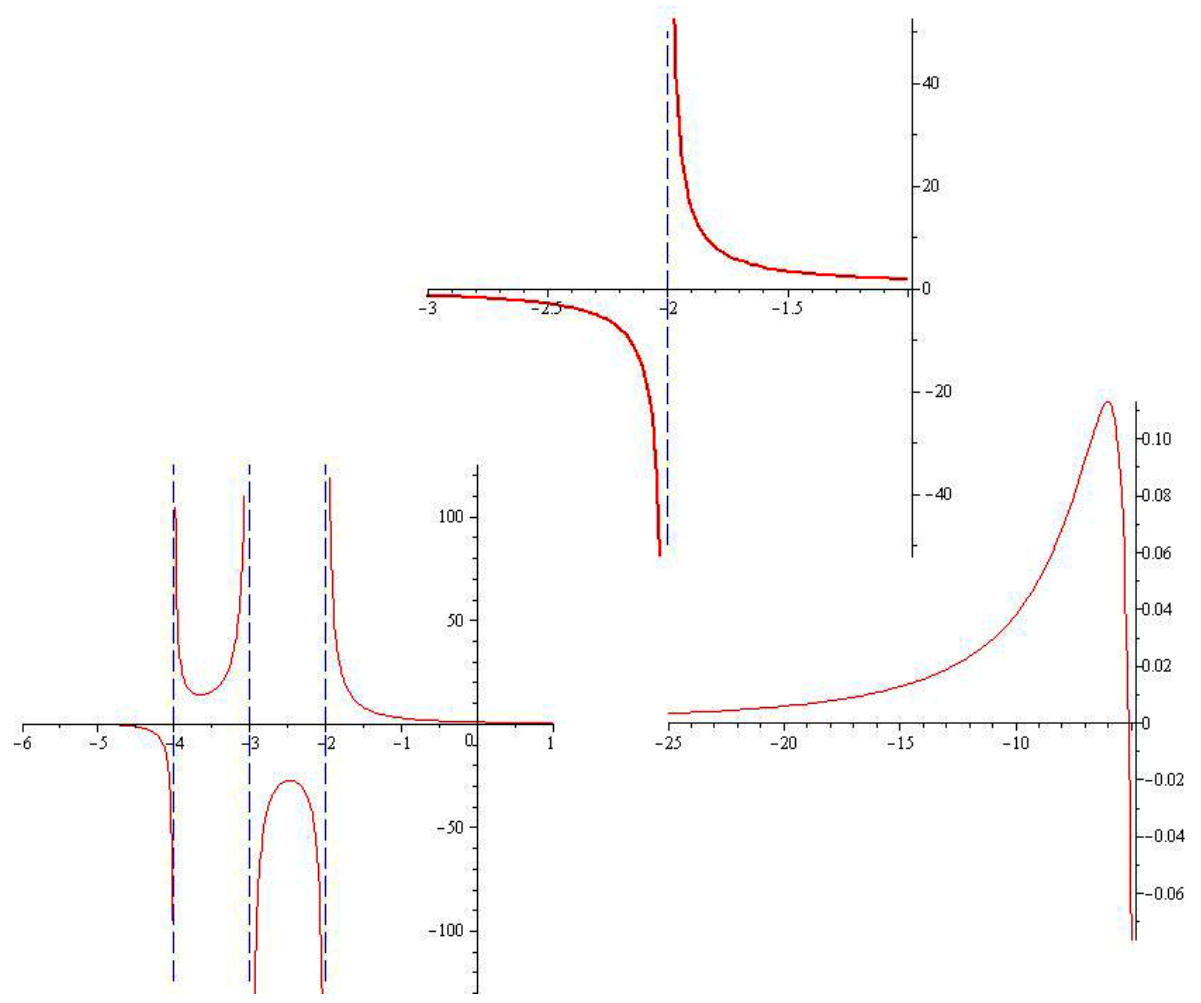

Figure 1: Analytic continuation: Plots of $B_{2}(s)$ (top) and $\Delta_{2}(s)$ (bottom) for real $s$ in various intervals. $B_{2}(s)$ is the expected value of $r^{s}$ where $r$ is distance from origin to a random point on the unit square $[0,1]^{2}$. Likewise, $\Delta_{2}(s)$ is the expectation with $r$ being the distance between two random points. $B_{2}(s)$ has a solitary pole at $s=-2$, while $\Delta(s)$ has three poles, at $s=-2,-3,-4$. Evidently, $\Delta_{2}(s)$ also has a mysterious zero at some real $s \approx-5.137 \ldots$ (see text). 